\title{
Independent movement of cusps during occlusal loading
}

\author{
R.L. Sakaguchi \\ E.W. Brust ${ }^{2}$ \\ M. Cross $^{3}$ \\ R. DeLong \\ W.H. Douglas'
}

'Biomaterials Research Center

University of Minnesota School of Dentistry

16-212 Moos Tower

515 Delaware Street, SE

Minneapolis, MN 55455

2Dept. of Orthodontics

University of Michigan

School of Dentistry

Ann Arbor, Ml 48109

${ }^{3}$ Centre for Numerical Modelling

\& Process Analysis

Thames Polytechnic

Wellington Street

London SE 18 6PF, England

\section{Received May 29, 1990}

Accepted January 18, 1991

This study was supported in part by USPHS

Research Grant T35 DE07098, Short-term

Training: Students in Professional Schools, from the National Institute of Dental

Research, Bethesda, MD 20892.

Dent Mater 7:186-190, July, 1991

Abstract-An integrated experimental and theoretical approach is proposed for the evaluation of stress within a natural tooth during bruxing. A physical model was developed that used strain gauges bonded to the buccal and lingual heights of contour of extracted, intact maxillary premolar teeth for the validation of a two-dimensional finite element model of a natural, intact maxillary premolar tooth. Results from the finite element model were in good agreement with the experimental results. The tooth exhibits "cuspal independence", which describes the relative independence of the loaded cusp from the remaining cusp that is not loaded. In other words, the control cusp does not demonstrate significant strain when the adjacent cusp is loaded. The overall stiffness of the tooth does not appear to be significantly affected by separation of the enamel of the two cusps and appears to be primarily a function of the dentin.
W hen an occlusal force and lateral excursion are generated between occluding surfaces, the friction between these surfaces causes stresses to be transmitted into the tooth and surrounding periodontal structures (Douglas et al., 1985). Most of these stresses are absorbed in the periodontal ligament, which deforms. However, static loading of the tooth has been shown to cause deformation of the crown of the tooth as well (Morin et al., 1988a; Sakaguchi, 1988). High occlusal loads, combined with the lateral excursive contacts seen in bruxism, may result in greater deformation of the crown.

Physical models can be used to show deformation under simulated bruxing conditions. The "Artificial Mouth", described in the literature (DeLong and Douglas, 1983, 1991), is capable of reproducing the parameters of mastication and, as such, is also able to simulate bruxing conditions. Generally, deformation of external surfaces is readily measured with bonded strain gauges. Measurement of strain at the internal aspects of the structure is more difficult. A mathematical model can represent the physical geometry of the structure of interest and, when appropriate loading and boundary conditions are used, the internal and external behavior of the structure can be studied. However, theoretical models require validation before the results can be interpreted with any degree of confidence. This important validation step can be accomplished through integration of straingauge methods and finite element analysis. Strain-gauge methods not only validate the results from the mathematical model, but also provide realworld boundary conditions that could otherwise only be speculative. Once the mathematical model has been validated, the solution can be extensively interrogated to provide the basis for a range of studies.

The integrated approach described has been used by Morin (1988a,b) to analyze the strain distribution of natural intact maxillary premolar teeth under centric occlusal loads with a number of different cavity preparations and restorations. Comparison of the results with those produced by the finite element method produced significant correlation. Strain gauges have also been used to study stress in other tooth-related areas (Buckland-Wright, 1978; Hylander, 1979). The finite element method (FEM) has been shown to produce results similar to those found by photo-elastic and strain-gauge studies (Hood et al., 1975; Farah et al., 1973; Kitoh et al., 1977).

The specific aims of this project were: (1) to evaluate the deformation of a natural premolar tooth during bruxing in an artificial mouth; and (2) to develop a validated and verified two-dimensional model of the stress-strain behavior of a premolar tooth and to use the mathematical model to provide information on the internal stress distribution during bruxing.

\section{MATERIALS AND METHODS}

Strain-gauge Measurement of Natural Tooth Deformation.-Four freshly extracted, intact maxillary second premolars were mechanically cleansed of soft tissue and debris, and stored in de-ionized water at $4^{\circ} \mathrm{C}$. Strain gauges (CEA-06-032UW-120, Measurements Group, Raleigh, NC) were positioned at the height of contour on the buccal and lingual surfaces of the tooth and bonded according to the manufacturer's methods (Fig. 1). The gauge and exposed wire ends were coated with light-cured Silux Enamel Bond Resin (3M Dental Products, St. Paul, MN). Pairs of teeth were mounted in nylon rings with clear Orthodontic Resin (L.D. Caulk, Milford, DE) apical to the cemento-enamel junction. Polyvinylsiloxane impression material (Express, 3M Dental Products, St. Paul, MN) was placed around the strain gauges and exposed wire ends for insulation from fluids. The system was placed into the mandibular member of the Artificial Mouth. The cuspal inclines were modified at the occlusal contact area to facilitate occlusal loading by a $4.4-\mathrm{mm}$ spherical loading element along the buccal and lingual triangular ridges.

A profile of the occlusal surface (buccolingual) was recorded and digitized before the loads were applied, so that the cuspal inclinations could be 
determined. Occlusal loads of $22 \mathrm{~N}, 35.2$ $\mathrm{N}$, and $44 \mathrm{~N}(5,8,10 \mathrm{lbs})$ were applied to the tooth in a bruxing pattern, starting at one cusp tip through centric occlusion to the opposite cusp tip and back. The strain gauges were connected to a Micro-Measurements 2100 System Strain Gauge Conditioner (Measurements Group, Raleigh, NC) via a half Wheatstone bridge and output to an XY recorder (Houston Instruments Omnigraphic 2000DX, Austin, TX). Strains were measured at the buccal and lingual heights of contour as a function of various occlusal forces and lateral excursion.

Development of the Finite Element Model.-The modeling phase of the study involved the development of a two-dimensional mathematical model representing the physical model. Two microcomputer-based finite element analysis packages were used: ANSYS PC/Linear (Swanson Analysis Systems, Inc., Houston, PA) and IFECS (Interactive Finite Element Computing System, Lewis and Cross) (Lewis and Cross, 1978).

ANSYS PC/Linear is an industry standard 2-D/3-D finite element analysis system. IFECS, written by Lewis and Cross (1978), is a two-dimensional $\mathrm{FE}$ software package that provides an interactive $\mathrm{PC}$ computing environment for FEM application. This includes preprocessing, automatic mesh generation, input data verification, and post-processing. An optimal triangular mesh is generated by use of the prescribed nodes as vertices, sorting out the relevant material properties for each element. Any errors incurred in definition of the boundary are determined by the mesh

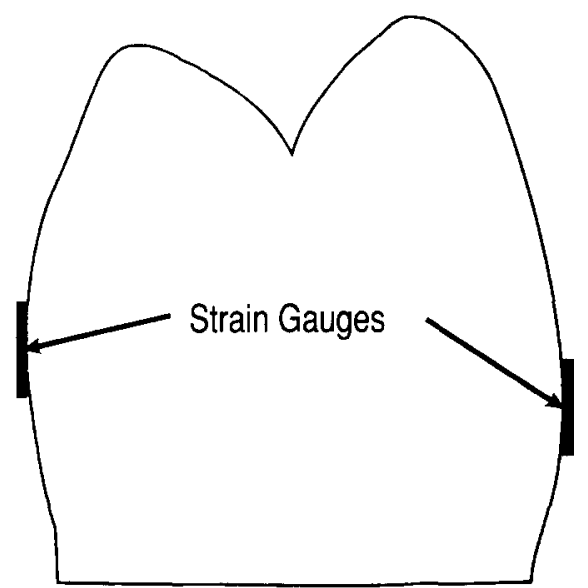

Fig. 1. A physical model of a natural premolar tooth with strain gauges attached at the buccal and lingual heights on contour. generation procedure. A CADD package (Generic Software, Redmond, WA) was used for node entry. Nodal points were modified in the ANSYS pre-processor, mesh generation was performed in IFECS, then ANSYS was used for solution and post-processing. A program was written to translate the files from one system to the other. The graphics post-processor for the $\mathrm{PC}$ version of IFECS was not complete at the time of this project; therefore, it was not used for solution or post-processing.

A model was constructed from the mean dimensions of a large population of natural teeth (Langsjoen and Noble, 1982) which represented an "average" maxillary first premolar. The two-dimensional plane strain model consisted of 343 nodes and 586 elements, with the eight nodes apical to the cemento-enamel junction fixed in the $\mathrm{X}$ and $\mathrm{Y}$ directions (Fig. 2). All other nodes were given two degrees of freedom (X and $\mathrm{Y}$ ). The material types and properties of the teeth, as described by Morin (1985) and Morin et ai. $(1988 \mathrm{a}, \mathrm{b})$, are listed in Table 1. Occlusal loads of $22 \mathrm{~N}, 35.2 \mathrm{~N}$, and $44 \mathrm{~N}$ $(5,8,10 \mathrm{lbs})$ were applied at discrete lateral excursive positions (six lingual, six buccal, and centric occlusion) to simulate bruxing in the experimental configuration (Fig. 3). The mean principal strain from four contiguous elements on the buccal and lingual heights of contour was compared with the straingauge results.

Several assumptions were made in the model:

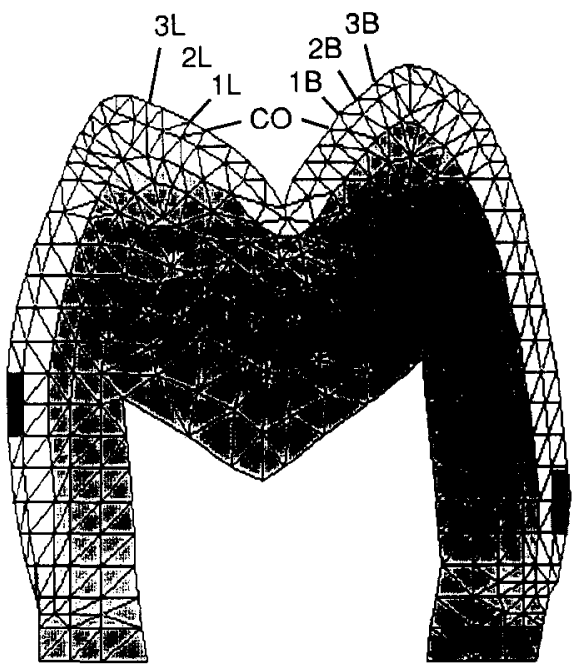

Fig. 2. Finite element model for a natural premolar. Shaded region indicates dentin with lighter-shaded enamel layer overlying. The shaded elements at the buccal and lingual heights of contour indicate the elements used to represent the strain-gauge positions. The position identifiers indicate positions which represent those on the physical model.
(1) absolute bonding between enamel and dentin,

(2) negligible effect of the pulp chamber on the performance of the model, and

(3) enamel and dentin modeled as isotropic, homogeneous materials.

A plane strain finite element model was implemented because of the assumption of uniform loading and uniform material properties in the tooth section of interest. The strain normal to the X-Yplane $\left(\varepsilon_{z}\right)$ and the shear strains $\left(Y_{\mathrm{xz}}\right.$ and $\left.Y_{\mathrm{Yz}}\right)$ are assumed to be zero. The assumption of plane strain is realistic for bodies with a constant crosssectional area along the $Z$ axis subjected to loads that act only in the $\mathrm{X}$ and/or $\mathrm{Y}$ directions and do not vary in the $Z$ direction. Only a unit thickness of the structure is considered because each unit thickness behaves identically (except near the ends). The behavior of the tooth crown at the mesial and distal marginal ridges was not of interest; therefore, this representation was appropriate.

\section{RESULTS}

The results from the experimental portion of the study are listed in Table 2. The columns represent strain-gauge readings at the buccal (B) or lingual (L) heights of contour on the premolar tooth for three different load applications (22 $\mathrm{N}, 35.2 \mathrm{~N}$, and $44 \mathrm{~N}$ ). The rows represent the travel of the maxillary loading element from a position on the buccal incline of the lingual cusp through centric occlusion to the incline of the buccal cusp, as shown in Fig. 3. The results are

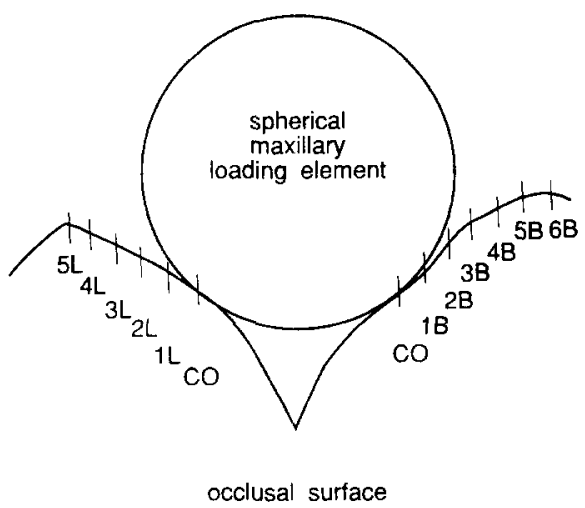

Fig. 3. Maxillary spherical loading element oriented on the occlusal surface with specific locations along the cuspal inclines identified to correspond to Table 2. CO = centric occlusion, IB $-6 \mathrm{~B}$ represent positions on the lingual incline of the buccal cusp, $1 \mathrm{~L}$ - 5L represent positions on the buccal incline of the lingual cusp. 


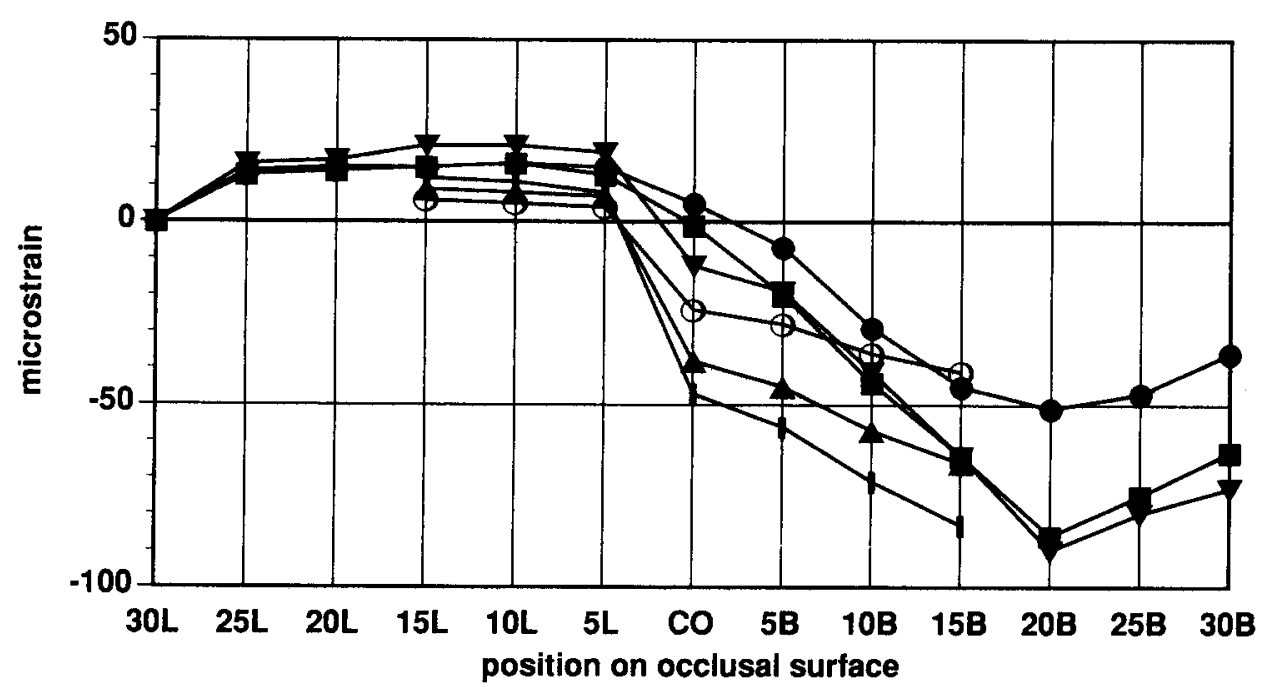

Fig. 4. Experimental and finite element analysis strain results for buccal gauge.
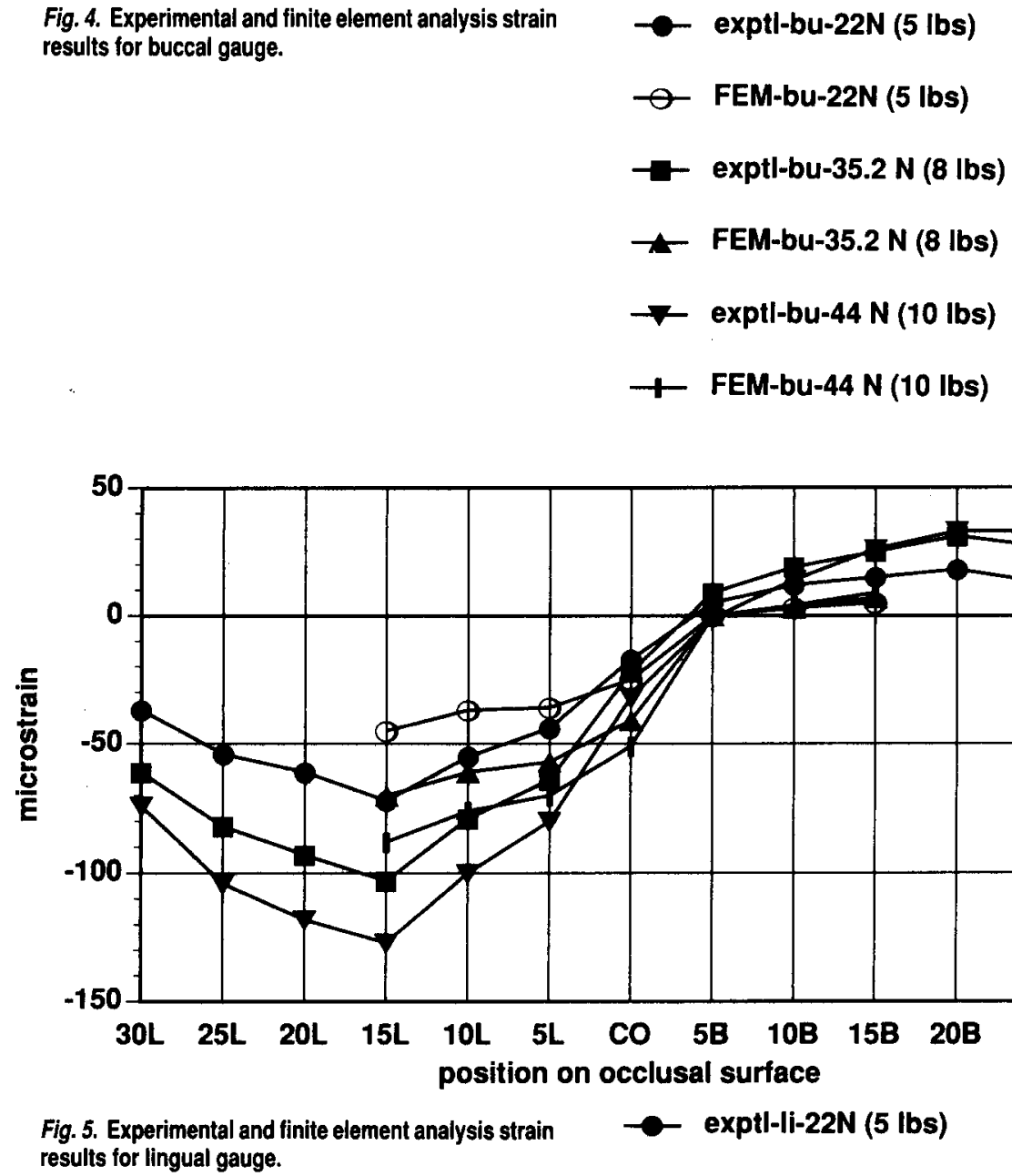
results for lingual gauge. reported in microstrain for each of the buccal and lingual strain gauges. The standard convention of representing tension by positive values and compression by negative values is used. Table 3 lists the results from the finite element computations, in the same format as Table 2. The experimental and finite element results are demonstrated graphically in Figs. 4 and 5.

For the experimental phase of the study, the strain generally increased on the loaded cusp as the lateral excursion increased,then leveled off as the loading element approached the cusp tip. The strain measured at the buccal and lingual strain gauges increased directly with the three applied load magnitudes for all lateral excursive positions. The cusp that was not loaded experienced small tensile strains which were generally $25 \%$ of the compressive strains seen on the loaded cusp. These characteristics were seen on both buccal and lingual cusps. The lingual cusp experienced greater deformation with the 44-N load than the buccal cusp under the same load.

The characteristic relationship between lateral excursion and strain was also seen in the finite element results. The strain passed through zero after the loading element moved from the centric occlusion location (Figs. 4 and 5).

\section{DISCUSSION}

The ability to alter structures and material properties under controlled conditions is one of the advantages of finite element analysis. The role of the integrity of the enamel shell was readily investigated with the finite element model, whereas experimental preparations would be difficult to control. However, it must be emphasized that theoretical models should be carefully validated by use of experimental techniques.

The two-dimensional finite element model produced good correspondence to the experimental result through accurate representation of the morphology and careful definition of boundary and loading conditions. Increasing the number of elements as a convergence test did not produce significant changes in the results.

An interesting result from this study, demonstrated by both the strain-gauge and the finite element methods, was the relative insensitivity of the cusp that was not loaded to the cusp that was loaded. As the one cusp was loaded, 


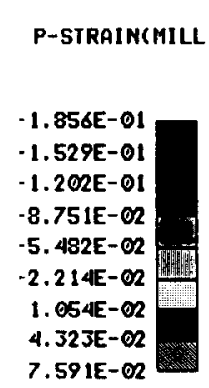

A

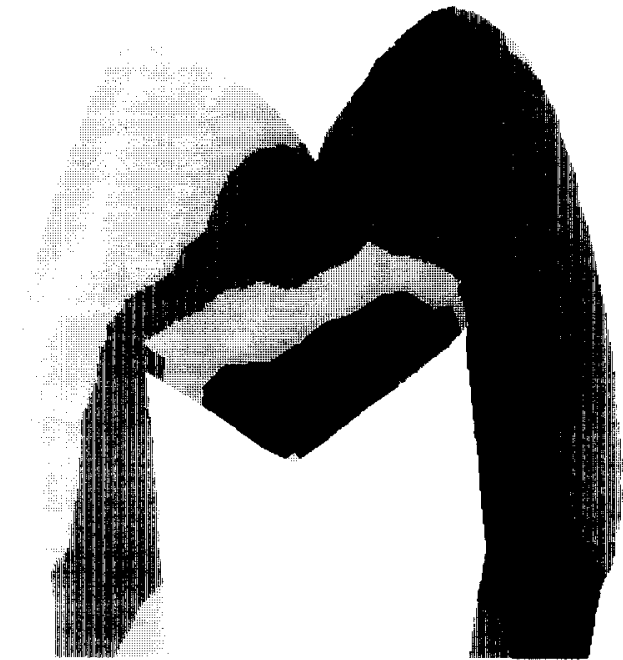

P-STRAIn(MILLI)

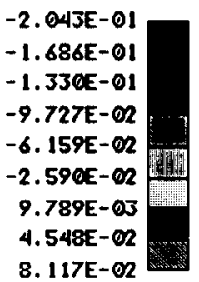

B

Fig. 6. Finite element models with intact enamel (A) and enamel separated at the central groove (B).

strains measured at the height of contour of the opposite cusp were low, indicating little deflection of the unloaded cusp. This phenomenon may be considered "cuspal independence". As part of a unit structure, it could be expected that the two cusps would function as an integrated unit, i.e., as the height of contour of the loaded cusp experienced compression, the unloaded cusp should follow this displacement and therefore experience tension (Tables 2 and 3; Figs. 4 and 5). However, the enamel that bridges the central fissure does not transmit the stress effectively between the cusps. The premolar tooth appears to behave as two independent singlecusped teeth that are attached at the central groove. Microscopic evaluation of the nature of the central fissure did not provide consistent evidence for the explanation of the independent movement of the cusps. The natural tooth performs in this manner because of the elasticity of the supporting dentin underlying the enamel and the intimate bond between the enamel and dentin which transmits the occlusal stress into the dentin. Occlusal stresses at one cuspal incline do not influence the adjacent cusp.

The enamel was separated at the central groove in the finite element model so that the effect of breaking the integ-

TABLE 1

MATERIAL PROPERTIES

\begin{tabular}{lll}
\hline Material & Poisson's Ratio & Mod. of Elast. $\left(\mathrm{N} / \mathrm{m}^{2}\right)$ \\
\hline Dentin & 0.25 & $1.54 \mathrm{E} 10$ \\
Enamel & 0.30 & $4.69 \mathrm{E} 10$ \\
\hline
\end{tabular}

rity of the outer enamel shell could be determined. When the enamel layer was parted down to the dentino-enamel junction separating the enamel on the buccal and lingual cusps, there was minimal effect on the deformation of the tooth crown under load (Figs. 6A and $6 \mathrm{~B})$. Elevated strains were noted at the base of the "fracture"; however, there was negligible effect on the rest of the tooth. This suggests that the overall deformation of the tooth is not so much a function of the integrity of the enamel in the central fissure as it is a function of the elastic properties of the dentin. The enamel adds to the stiffness of the

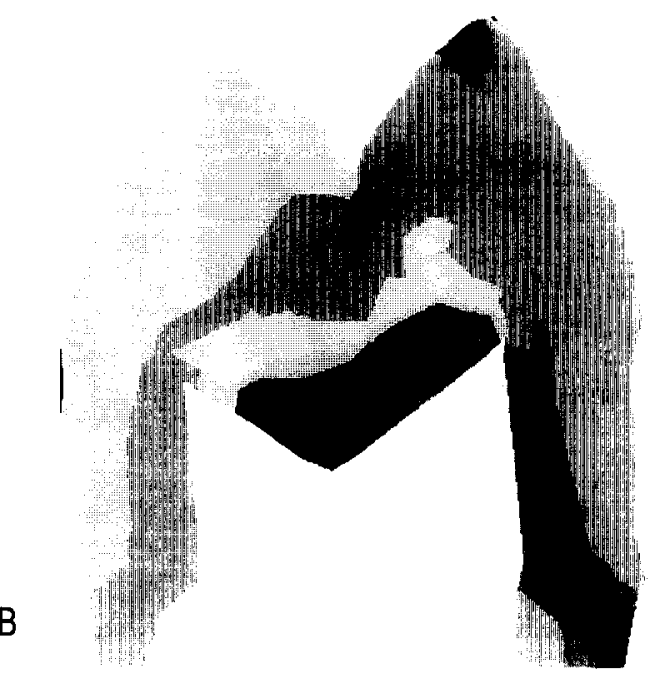

structure, but does not dictate whether the tooth exhibits cuspal independence. Therefore, it is important for as much dentin as possible to be maintained in restorations, so that the tooth can deform as a natural, intact tooth. Perhaps a greater loss of enamel is not as detrimental as the loss of large amounts of dentin.

Bruxing loads were applied in this study to produce worst-case estimates for the deformation of the tooth crown. Bruxing loads are particularly damaging because of the high occlusal loads and persistent occlusal contacts. This long stroke results in significant strains

TABLE 2

EXPERIMENTAL STRAIN GAUGE MEASUREMENTS (in microstrain)

\begin{tabular}{lllllll}
\hline \multirow{2}{*}{ Strain Gauge Location: } & $\mathrm{Li}$ & $\mathrm{Li}$ & $\mathrm{Li}$ & $\mathrm{Bu}$ & $\mathrm{Bu}$ & $\mathrm{Bu}$ \\
\cline { 2 - 7 } Load: & $22 \mathrm{~N}$ & $35.2 \mathrm{~N}$ & $44 \mathrm{~N}$ & $22 \mathrm{~N}$ & $35.2 \mathrm{~N}$ & $44 \mathrm{~N}$ \\
\hline${ }^{3} 30 \mathrm{~L}$ & -37 & -61 & -74 & 0 & 0 & 0 \\
$25 \mathrm{~L}$ & -54 & -82 & -104 & 14 & 13 & 16 \\
$20 \mathrm{~L}$ & -61 & -93 & -118 & 15 & 14 & 17 \\
$15 \mathrm{~L}$ & -72 & -103 & -127 & 15 & 15 & 21 \\
$10 \mathrm{~L}$ & -55 & -79 & -100 & 16 & 16 & 21 \\
$5 \mathrm{~L}$ & -44 & -64 & -80 & 15 & 13 & 19 \\
$\mathrm{CO}$ & -17 & -22 & -32 & 5 & -1 & -12 \\
$5 \mathrm{~B}$ & 5 & 9 & -1 & -7 & -20 & -19 \\
$10 \mathrm{~B}$ & 12 & 19 & 14 & -29 & -44 & -41 \\
$15 \mathrm{~B}$ & 15 & 25 & 26 & -45 & -64 & -64 \\
$20 \mathrm{~B}$ & 18 & 31 & 33 & -51 & -86 & -90 \\
$25 \mathrm{~B}$ & 13 & 27 & 33 & -47 & -75 & -80 \\
$30 \mathrm{~B}$ & -4 & -2 & -2 & -36 & -63 & -73
\end{tabular}

"Position of the maxillary loading element moving from the buccal incline of the lingual cusp (L) through centric occlusion (CO) to the lingual incline of the buccal cusp (B). See Fig. 3. 
at the buccal and lingual heights of contour. The natural tooth is able to disperse the occlusal stresses into the dentin, thus minimizing damage to the tooth. However, the restored tooth does not have the benefit of absolute bonding and stress transfer to the dentin. Under bruxing stresses, it is not uncommon to find fractured restorations and failure at the restoration/natural tooth interface.

The finite element model produced significant correlation with the results from the experimental phase of the study ( $\mathrm{r}>0.95$ ). The characteristic cuspal independence, as well as the quantitative results, were in good agreement. The finite element model demonstrated maximum compressive strains directly under the occlusal loads, corresponding to the findings of Selna et al. (1975). The intimate bond of the dentino-enamel junction enables the natural tooth to distribute these compressive stresses into the elastic dentin layer. This tends to minimize the damage from local stresses on the enamel that result from occlusal contacts.

Further studies comparing the stress distribution between full-coverage restorations and the natural tooth will follow.

\section{CONCLUSIONS}

A two-dimensional finite element model of a natural, intact maxillary premolar tooth was developed to correspond to the external behavior of a physical model. The finite element model was validated by measurement of the strain on the physical model with strain gauges located at the buccal and lingual heights of contour.

Both the physical and the computational models demonstrated cuspal independence (i.e., the cusps moved with relative independence when loaded). Separating the enamel at the central groove did not significantly alter the deformation of the tooth crown during occlusal loading. Cuspal independence is attributed to the elastic behavior of dentin and the stress transfer capabilities of the dentino-enamel junction.

\section{REFERENCES}

BuckLAND-Wright, J.C. (1978): Body Structure and Pattern of Force Transmission in the Cat Skull, $J$ Morphology 155:35-62.

TABLE 3

FINITE ELEMENT COMPUTATION RESULTS (in microstrain)

\begin{tabular}{lllllll}
\hline Location: & $\mathrm{Li}$ & $\mathrm{Li}$ & $\mathrm{Li}$ & $\mathrm{Bu}$ & $\mathrm{Bu}$ & $\mathrm{Bu}$ \\
\cline { 2 - 7 } & $22 \mathrm{~N}$ & $35.2 \mathrm{~N}$ & $44 \mathrm{~N}$ & $22 \mathrm{~N}$ & $35.2 \mathrm{~N}$ & $44 \mathrm{~N}$ \\
\hline
\end{tabular}

*30L

$25 \mathrm{~L}$

$20 \mathrm{~L}$

$\begin{array}{rllllll}15 \mathrm{~L} & -45 & -70 & -88 & 6 & 9 & 12 \\ 10 \mathrm{~L} & -37 & -61 & -76 & 5 & 8 & 11 \\ 5 \mathrm{~L} & -36 & -57 & -70 & 4 & 7 & 8 \\ \mathrm{CO} & -25 & -41 & -51 & -24 & -38 & -47 \\ 5 \mathrm{~B} & 0 & 0 & 0 & -28 & -45 & -56 \\ 10 \mathrm{~B} & 3 & 3 & 4 & -36 & -57 & -71 \\ 15 \mathrm{~B} & 5 & 7 & 9 & -41 & -66 & -83\end{array}$

20B

$25 B$

"Simulation of the maxillary loading elementmoving from the buccal incline of the lingual cusp (L) through centric occlusion (CO) to the lingual incline of the buccal cusp (B). See Fig. 3.

DeLong, R. and Douglas, W.H. (1983): Development of an ArtificialOral Environment for the Testing of Dental Restoratives: Bi-axial Force and Movement Control, J Dent Res 62:3236.

DeLong, R. and Douglas, W.H. (1991): An Artificial Oral Environment for Testing Dental Materials, IEEE Trans Biomed Eng 38:339-345.

Douglas, W.H.; Sakaguchi, R.L.; and DeLong, R. (1985): Frictional Effects Between Natural Teeth in an Artificial Mouth, Dent Mater 1:115-119.

FARAH, J.W.; Craig, R.G.; and Sikarski, D.L. (1973): Photoelastic and Finite Element Stress Analysis of a Restored Axi-Symmetric First Molar, $J$ Biomechan 6:511-520.

HoOD, J.A.; FARAH, J.W.; and Craig, R.G. (1975): Modification of Stresses in Alveolar Bone Induced by a Tilted Molar, J Fixed Prosthet 34:415-421.

Hylander, W.L. (1979): Mandibular Function in Galago crassicaudatus and Macaca fascicularis: An in vivo Approach to Stress Analysis of the Mandible,JMorphology 159:253-296.

KITOH, M.; SUETSUGU, T.; and MURAKAMI, Y. (1977): Mechanical Behavior of Tooth, Periodontal Membrane and Mandibular Bone by the Finite Element Method, Bull Tokyo Med Dent Univ 24:81-87.
LANGSJOEN, O.M. and NOBLE, F.W.(1982): Descriptive Dental Anatomy, Minneapolis: University of Minnesota.

Lewis, B.A. and Cross, M. (1978): IFECS-An Interactive Finite Element Computing System, Appl Math Modelling 2:165-176.

Morin, D.L. (1985): Relationship Between Cavity Form, Restorative Technology and Strength ofRestored Teeth, Master of Philosophy Thesis, London: Thames Polytechnic.

Morin, D.L.; Douglas, W.H.; Cross, M.; and DeLong, R. (1988a): Biophysical Stress Analysis of Restored Teeth: Experimental Strain Measurement, Dent Mater 4:41-48.

Morin, D.L.; Cross, M.; Voller, V.R.; Douglas, W.H.; and DeLong, R. (1988b): Biophysical Stress Analysis of Restored Teeth: Modelling and Analysis, Dent Mater 4:77-84.

SAKAGUCHI, R.L. (1988): A Biophysical Analysis of the Occlusal Wear of Dental Materials, PhD Thesis, London: Thames Polytechnic.

Selna, L.G.; Shillingburg, H.J.; and KERR, P.A. (1975): Finite Element Analysis of Dental Structures-Axisymmetric and Plane Stress Idealizations, JBiomed Mater Res 9:237252. 